\title{
Neuroparacoccidioidomycosis with concomitant pulmonary and vocal cord lesions
} Neuroparacococcidioidomicose com lesões concomitantes nos pulmões e na corda vocal Adriano Basso Dias' ${ }^{1}$ Cláudia Scherber Giugno², Jessica Oliboni Scapineli³, Gabriel Pedro Tarso ${ }^{3}$, Rodrigo Miranda de Curtis ${ }^{1}$, Rene Lenhardt ${ }^{1}$

Paracoccidioidomycosis is a systemic granulomatous disease caused by the fungus Paracoccidioides brasiliensis ${ }^{1}$. Involvement of the central nervous system is more common in the chronic form of the disease, affecting the supratentorial region in two-thirds of cases ${ }^{2}$. A previously healthy 48-yearold woman presented with a two-month history of headache, cough and hoarseness. Brain magnetic resonance imaging revealed multiple ring enhancing cerebellar lesions (Figure 1), most of them with imaging features indicative of granulomas probably with liquefied necrosis. (Figure 2). Chest CT showed scattered nodules and cavitations (Figure 3). Fiber bronchoscopy identified a vocal cord lesion, which was biopsied. The histopathological findings were compatible with paracoccidioidomycosis (Figure 4).
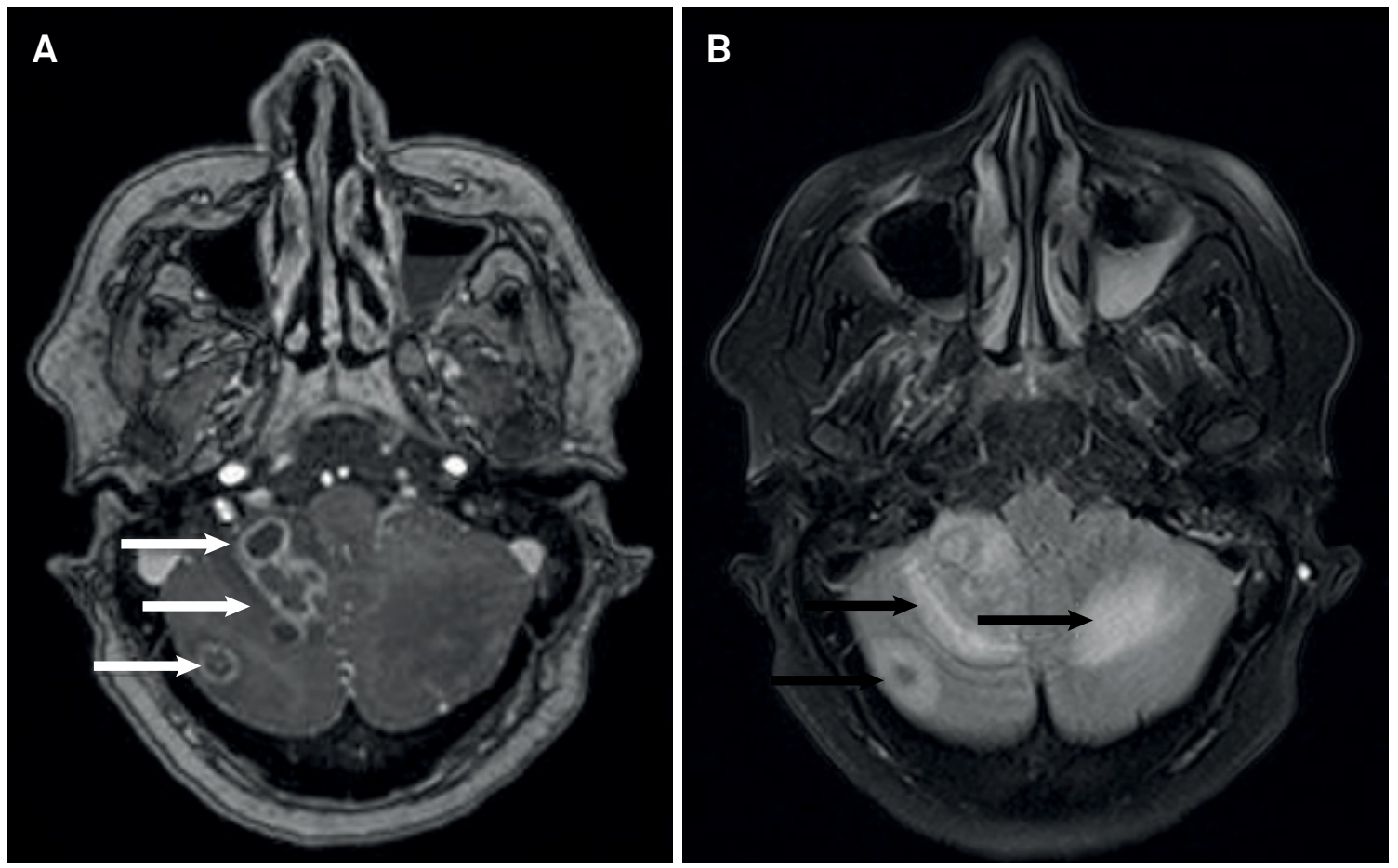

Figure 1. Brain magnetic resonance. A) Axial postcontrast T1-weighted image shows multiple cerebellar lesions with ring enhancement (white arrows). B) Axial FLAIR image demonstrates that these lesions present variable signal intensities, associated with moderate surrounding vasogenic edema (black arrows).

\footnotetext{
${ }^{1}$ Hospital Dom Vicente Scherer, Irmandade Santa Casa de Misericórdia de Porto Alegre, Departamento de Radiologia e Diagnóstico por Imagem, Porto Alegre RS, Brasil;

${ }^{2}$ Irmandade Santa Casa de Misericórdia de Porto Alegre, Departamento de Patologia, Porto Alegre RS, Brasil;

${ }^{3}$ Irmandade Santa Casa de Misericórdia de Porto Alegre, Departamento de Medicina Interna, Porto Alegre RS, Brasil.

Correspondence: Rene Lenhardt; Departamento de Radiologia e Diagnóstico por Imagem, Hospital Dom Vicente Scherer, Irmandade Santa Casa de

Misericórdia de Porto Alegre; Av. Independência, 75; 90020-160 Porto Alegre RS, Brasil; E-mail: reneneuro@gmail.com

Conflict of interest: There is no conflict of interest to declare.

Received 29 May 2018; Received in final form 05 July 2018; Accepted 29 July 2018.
} 


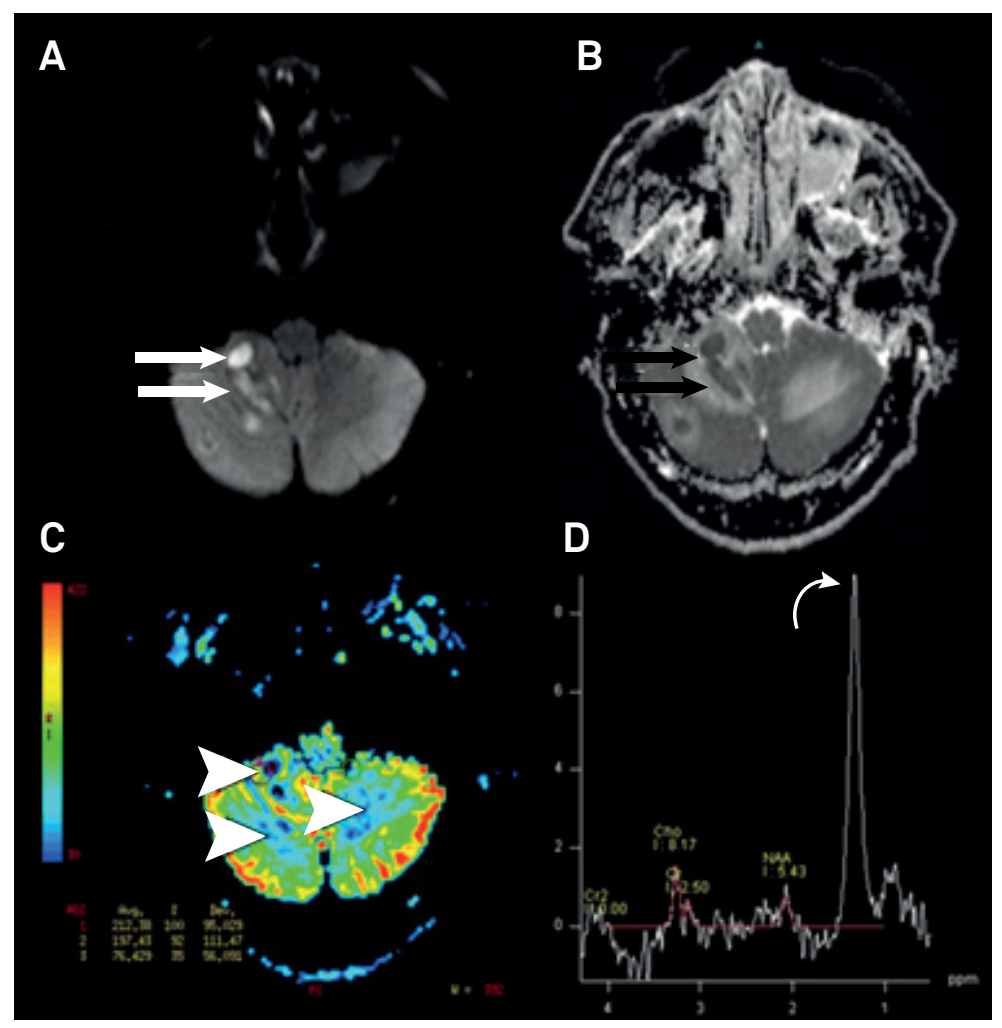

Figure 2. A) Diffusion-weighted imaging demonstrates that most of lesions are hyperintense (straight white arrows). B) Apparent diffusion coefficient map shows hypointensity in these lesions, compatible with restricted diffusion (straight black arrows). C) Magnetic resonance perfusion reveals decreased perfusion in the lesions and adjacent white matter (arrowheads). D) Magnetic resonance spectroscopy, single voxel, echo time $=35 \mathrm{~ms}$, shows elevation of lipids and/or lactate peaks (curved arrow). The N-acetylaspartate peak is decreased (signaling neuroaxonal loss or dysfunction). These findings are suggestive of granulomatous lesions, probably with liquefied necrosis.
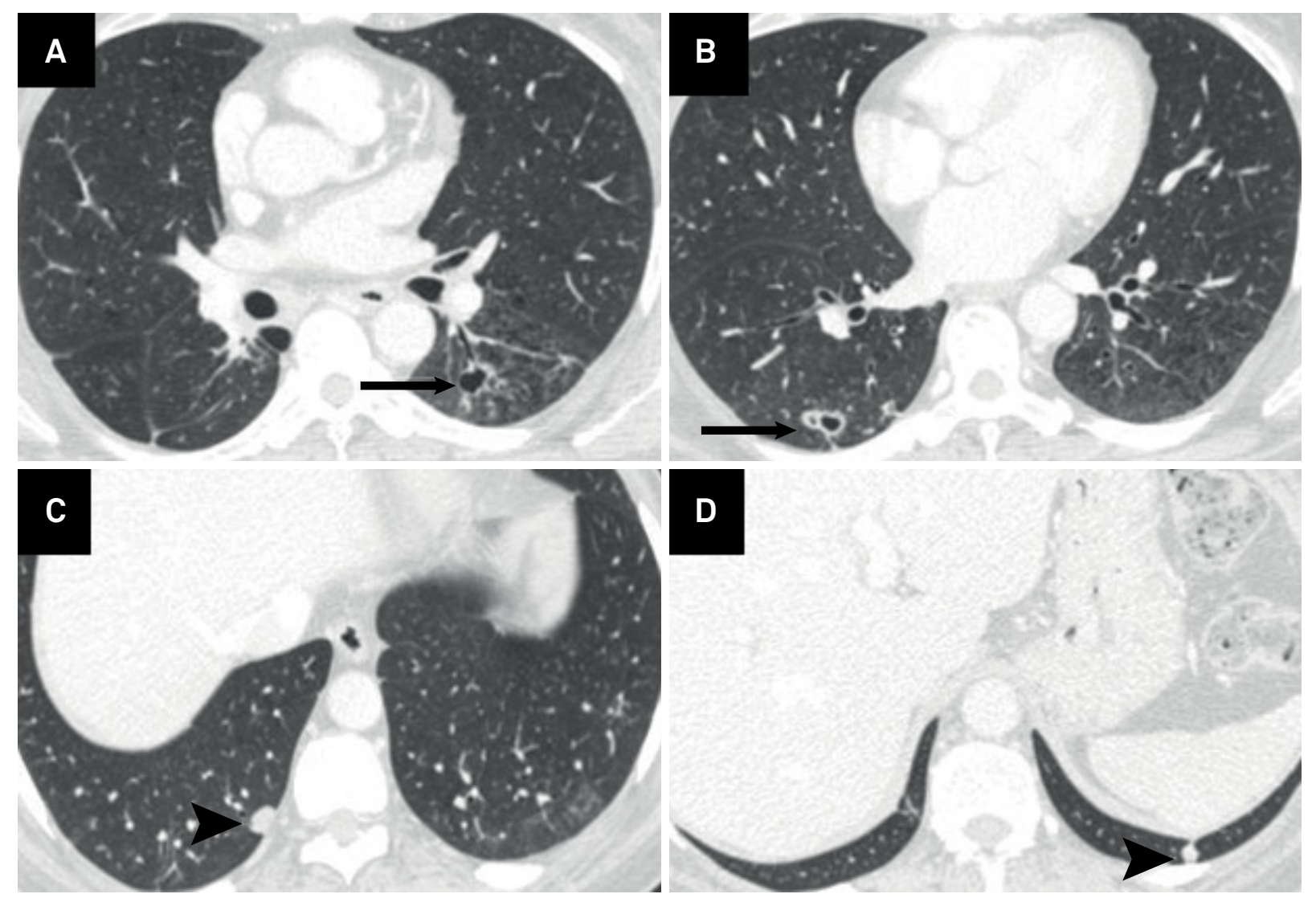

Figure 3. A-D: Axial chest computed tomography reveals scattered nodules (arrowheads) and cavitations (arrows), suggesting the possibility of a granulomatous process. 

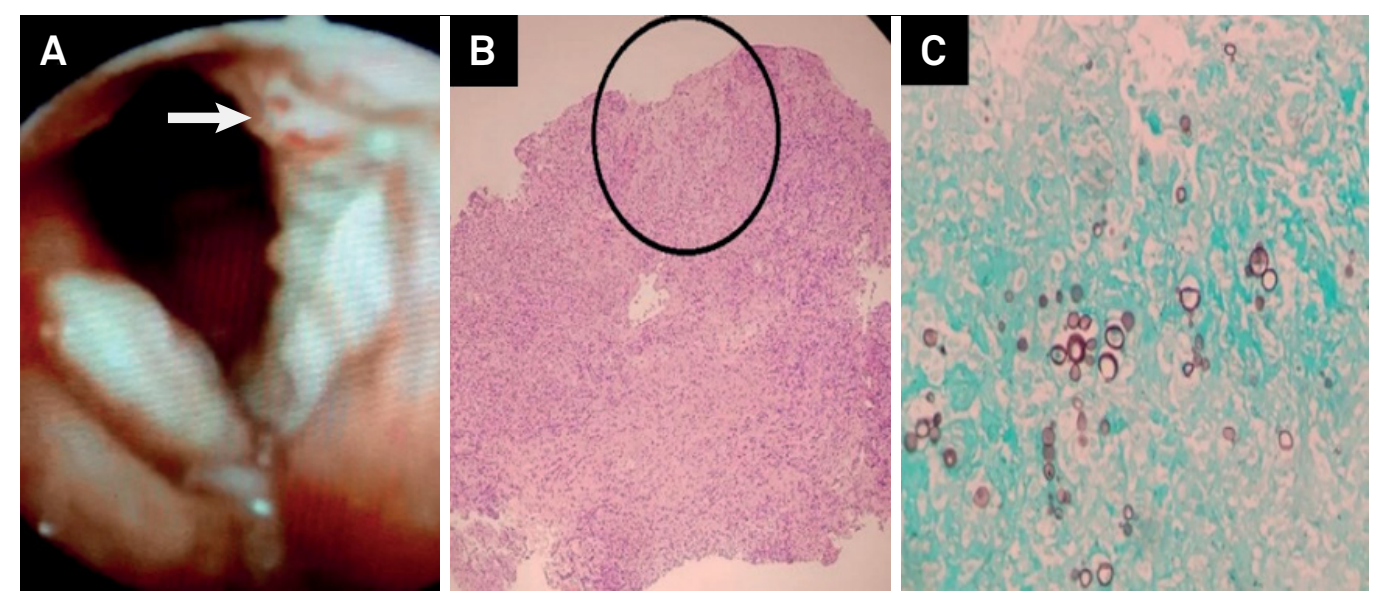

Figure 4. A) Fiber bronchoscopy reveals a vocal cord lesion (arrow), which was biopsied. B) Histological evaluation (H\&E, 10x) shows focus of granulomatous inflammation (inside the circle). C) Histological evaluation (Grocott stain, 400x) demonstrates fungal pathogens, with characteristic yeast elements with multipolar budding of variable size, compatible with Paracoccidioides brasiliensis.

\section{References}

1. Isolan GR, Vieira DM, Hehn F, Antunes AC. Paracoccidioidomycosis simulating brain tumor. Surg Neurol Int. 2014 Sep;5:134. https://doi.org/10.4103/2152-7806.140656
2. Pedroso VS, Vilela Mde C, Pedroso ER, Teixeira AL.

Paracoccidioidomycosis compromising the central nervous system: a systematic review of the literature. Rev Soc Bras Med Trop. 2009 Dec;42(6):691-7. https://doi.org/10.1590/S0037-86822009000600016 